\title{
Review on COVID-19 Post-Pandemic Emergencies in Health Sectors
}

\author{
Omer Fayshal Pavel ${ }^{1 *}$, Md. Omar Faruk Parvez ${ }^{2}$, S. M. Shahnawaz Rashid ${ }^{3}$ \\ ${ }^{1}$ Department of Pharmacy, East West University, Bangladesh \\ ${ }^{2}$ Medical Officer, Directorate General of Health Services, Bangladesh \\ ${ }^{3}$ Department of Pharmacy, University of Asia Pacific, Bangladesh
} *Address for Correspondence: Omer Fayshal Pavel, Graduate, Department of Pharmacy, East West University, Dhaka,
Bangladesh

E-mail: o.f.pavelewu@gmail.com

Received: 14 Mar 2020/ Revised: 30 May 2020/ Accepted: 19 Aug 2020

\begin{abstract}
COVID-19 was declared as global pandemic on the $11^{\text {th }}$ of March, 2020 and until mid of August more than 2 million people got infected with COVID-19. Most of the countries are under lockdown and having a major focus on developing vaccine to stop this nightmare. In this extreme time hospitals with other health facilities are under extreme pressure to eradicate the COVID-19 threat, meanwhile previously highly concerning health issues like NCDs, Changing in Microbial patterns including gut microbes, fear or anxiety and other mental health disturbances are emerging silently. Those may raise massive hazards just after the COVID19 pandemic. In this review paper, some health issues that may create an alarming situation in post-pandemic time are discussed briefly.
\end{abstract}

Key-words: COVID-19, Gut Microbe, Mental health, Non-Communicable Diseases, Post Pandemic, Resistant Bacteria

\section{INTRODUCTION}

COVID-19, viral flu caused by SARS-CoV-2 virus has created the global pandemic ${ }^{[1]}$. SARS-CoV-2 is a single strand RNA virus ${ }^{[2]}$, first detected in Wuhan, China in early December 2019. This viral flu has symptoms of fever, sore throat, cough, fatigue, headache ${ }^{[3]}$. In some cases, nausea or vomiting, diarrhoea, loss of taste and smell are recorded ${ }^{[4-6]}$. It was declared as global pandemic on the $11^{\text {th }}$ of March 2020 by World Health organization ${ }^{[9]}$. The virus damages several organs in the body by binding the ACE2 receptors ${ }^{[7]}$. The major targeting part of the virus is the cells of lungs and causes the death of the lung cells resulting in severe breathing problem. Moreover, by targeting gastrointestinal tracts that causes abdominal discomfort and diarrhoea ${ }^{[8]}$. Till mid of August'20, the globally total number of the infected case was more than 20 million and death was 741 thousand ${ }^{[10]}$.

\section{How to cite this article}

Pavel OF, Parvez OF, Rashid SMS. Review on COVID-19 PostPandemic Emergencies in Health Sectors. SSR Inst. Int. J. Life Sci., 2020; 6(5): 2646-2651.

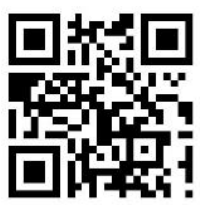

To reduce the spreading of the deadly virus several measures are taken like, keeping physical and social distancing, often washing hands with soap or using alcohol-based sanitizer, wearing masks that help to stop the spreading the virus ${ }^{[11]}$.

The major step taken by almost all the government authorities in many countries is 'Lockdown'. That means, without very emergency people are not allowed to move out. As a result, several organizations were not able to run properly due to the lack of human resource that leads to the economic crisis. According to the International Labor Organization (ILO) currently, more than 1 billion of workers are at high risk of salary reduction or losing their job and till now globally Gross Domestic Product growth reduced 6\% ${ }^{[12]}$.

However, the economy is not the only one that is impaired by COVID-19 but there are so many health services curtailed in the provision of COVID-19. Moreover, some disease and future alarming health issues like Non-Communicable Diseases (NCDs) and management of chronic disease, changes in microbiome patterns including the gut microbes, mental health degradation, managing the side effects of unnecessary taken medicines. Previously the pandemic of 1917/18, just after the pandemic there were several problems. 
Besides the low income, reduction in educational attainment and the physical disabilities had also increased significantly ${ }^{[13]}$.

In this paper, the discussion will be on the direct health issues that will be threatened in the post-pandemic time, just after COVID-19 is over. In this segment, the notables are antimicrobial resistance and gut microbial patterns, management of NCDs and chronic diseases. Another concerning issue in a post-pandemic mental health crisis and side effects of notoriously recommended medicines for the treatment of COVID-19.

This review article is carried out by assessing 28 articles and some websites that are sharing authentic and relevant information. Most of the articles are regarding COVID-19 or, the information on individual challenges of post-pandemic time. Assessing articles were selected and searched in PMC and Google scholars; most of the articles were from the different segments of journals like clinical neuropathy, anxiety disorders, infectious diseases, clinical microbiology, non-communicable disease and gastroenterology related journals. There were no location-based criteria on selecting articles rather the major focus was given on the topic of the article. The web links were selected by assuring the authenticity of the source. Most of the referred web sites are websites of WHO, NIHS, worldometers.info and some others.

Changes in Microbial Pattern and Effects on Gut Microbes- In COVID-19, the most recommended thing is to wash hands and regularly used materials with detergent, antiseptics or other cleaning products, which is very important to stay safe from the SARS-CoV-2 virus. But overuses or misuse of any product causes adverse effects. Overuse of the cleansing products may have dramatic effects on the environmental microbial system ${ }^{[14]}$. It is important to know that the cleansing products can't discriminate between the good and bad microbes. Additionally, several studies have shown that minimum inhibitory concentration (MICs) of anti-microbial agents can induce horizontal transfer of genes that are responsible for antibiotic resistance ${ }^{[15]}$. A theory named 'Hygiene Hypothesis' indicates that though sanitization or use of antibiotic make us safe from infections it also plays a role in developing several allergic diseases ${ }^{[16]}$. Another study finds a relationship between the reduction of the gut microbes and prevalence of NonCommunicable Diseases (NCDs), where NCDs may

increase by reduction of these gut microbes ${ }^{[17]}$. A specific example of the chemical Triclosan, which is used in many antiseptic preparations like detergents, hand sanitizers and soap, is absorbed by the skin ${ }^{[14]}$. Long time exposure to Triclosan may result in endocrine disorders, colonic inflammations and antibiotic resistance [18]. Another statement by Canadian Healthy Infant Longitudinal Development (CHILD) has shown that more frequent use of antiseptic may increase the amount of Lachnospiraceae, which is responsible for childhood obesity and overweight ${ }^{[19]}$. On the other hand, $A$ in vitro study provides weak causal evidence to attribute antimicrobial resistance to biocide usage ${ }^{[20]}$. The major classes of disinfectants and sanitizers are iodophors, quaternary ammonium compounds (QAC), peroxides, phenols, chlorine, and glutaraldehyde products. Bacterial resistance to each of these has been found. Disinfectants and sanitizers resistance can have a significant impact o health and economic consequences. Moreover, we have already a list published by WHO for urgently required concern of R\&D to develop new antibiotics (Table 1) ${ }^{[33]}$. However, the mechanisms of resistance have received uneven attention. Even the frequency with which resistance develops and the impact of environmental factors on resistance development is still less known. Coresistance of disinfectant-resistant bacteria to antibiotics occurs as a result of QAC resistance genes being located on transmissible plasmids and within conserved regions, and each of which has been shown to carry multiple antibiotic resistance genes ${ }^{[21]}$.

Table 1: List of WHO priority pathogens for the development of new antibiotics ${ }^{[33]}$

\section{Priority 1: Critical}

Acinetobacter baumannii

Pseudomonas aeruginosa

Carbapenem-resistant

Enterobacteriaceae

\section{Priority 2: High}

Enterococcus faecium

Staphylococcus aureus

Helicobacter pylori

Campylobacter sp.

\section{Vancomycin-resistant}

Methicillin-resistant

Vancomycin-

intermediate and resistant

Clarithromycin-resistant

Fluoroquinoloneresistant 
Salmonellae

Neisseria gonorrhoeae

Fluoroquinoloneresistant

Cephalosporin-resistant

Fluoroquinoloneresistant

\section{Priority 3: Medium}

Streptococcus pneumonia Penicillin-non-susceptible

Haemophilus influenzae

Shigella sp.

Ampicillin-resistant

Fluoroquinolone-

resistant

Apart from the resistance issue, the pandemic hampers the major surgical interventions of gastrointestinal tracks. For example, endoscopy is one of that. The American Society of Gastrointestinal Endoscopy (ASGE) and European Society of Gastrointestinal Endoscopy (ESGE) have recommended gastrointestinal obstruction needs emergency decompression and should be done even in the pandemic with proper precautions. Before starting the diagnostic process, risk assessment of COVID-19 must be done that may take additional 1-2 days before the surgery. One point should be noted that almost $53.4 \%$ of patients of COVID-19 has SARS-CoV-2 virus is the faeces sample, which indicates that the virus can travel in the $\mathrm{Gl}$ tract. These issues are taking more concern before going for the surgical or diagnostic procedure on any adverse events of GI track and thus patients with COVID-19 may produce risk for the surgeon if proper precautions are not taken. The major problem in endoscopy will be just after pandemic with the long waiting list of previously cancelled patients ${ }^{[22]}$.

\section{Non-Communicable diseases (NCDs) and Chronic} Diseases- COVID-19 has crucially curtailed the maintenance of healthcare service of non-communicable diseases (NCDs) in 155 countries over three weeks in May stated by the World health organization (WHO) ${ }^{[23]}$. The normal scenario of NCDs, it kills almost 41 million people in last year and which caused $71 \%$ of total death globally. Among this number about 15 million was aged between 30 to 69 and $85 \%$ of this unexpected death was in the lower and middle-income countries. The survey of WHO says that $94 \%$ of the responding countries had reassigned the health ministry staff from work on NCDs to dealing with the massive pandemic. As a result Hypertension treatment has been disrupted $49 \%$, cancer treatment $42 \%$ and the same in cardiovascular emergency responses in $31 \%$ of the countries. As an example, the Netherlands, new diagnosis of cancer have fallen $25 \%$ since the pandemic began. In Asian countries, the scenario is the same as in rural areas of India $30 \%$ of cardiac emergencies has reached in the health facilities. A statement of WHO indicated that post-pandemic health services will need an NCD plan to regain the ground lost ${ }^{[24]}$.

In the past three decades, a major effort has been given to deal with these chronic diseases. Many of the risk factors for cancer, heart disease, stroke and others have been identified. This is mostly where economic conditions have improved with major industrialization. Diabetes and cardiovascular disease had reached alarming proportions. Type 2 Diabetes is now emerging as the new pandemic of the 21st century. It is estimated that globally 150 million people will suffer from diabetes, with that number expected to double by $2025^{[25]}$.

Mental Health (Fear and Anxiety)- Mental health, especially fear and anxiety are the major issues that must be given concern in post-pandemic time. In the majority of the cases, the self-isolation or financial hardship are the major sources of the anxiety. However, it can't be ignored that the fear of coronavirus is also an emerged issue in the pandemic which also producing issues like mood disorders, anxiety and Post Traumatic Stress Disorders (PTSD) in many people. In a current pandemic, it is estimated that $10 \%$ of the total population will grow severe psychological problems and which will be a matter of severe concern in the postpandemic era ${ }^{[26,27]}$. A term Xenophobia, that is fear of the people coming from outside may have the COVID-19 infection or COVID stress syndrome that results in the fearful nightmare or intrusive thoughts of COVID are also playing a vital role to produce the mental health disturbance ${ }^{[28]}$, like the fear that short-lived response or anxiety is not short-lived one ${ }^{[30]}$.

There is a model proposed by Schimmenti, Billieus and Starcevic in 2020 that makes a time demanding framework. It states the fear can be four types in this situation that may produce mental vulnerability first, the physiological fear that denotes the fear of getting infected by COVID-19, then interpersonal fear represents that the fear for the nearer or closer person of getting infected. Third, Cognitive fear that means fear of not knowing or in some cases knowing the COVID-19 related updates or destructive information. Lastly, the fear in the behavioural part that is, fears of taking action. ${ }^{[29]}$. 
This mental health problem is not only for the general people it may also affect the caregiver, means doctor, nurses, medical technicians to volunteers all. COVID-19 put the healthcare professionals under in unpredicted and extreme work pressure. As they are getting more connected to the patients thus are more vulnerable to get infected by the virus. In many caregivers, especially medical students some negative thoughts may come like thinking himself as a terrible person or might be thought his supervisor doesn't care about his life because of doing duty in the COVID unit ${ }^{[31]}$. Once the pandemic moves away healthcare stuff must be actively monitored and supported. The National Institute for Health and Care Excellence recommended 'Active Monitoring' of staff to ensure the proper mental health sustainability and to assess the evidence-based care ${ }^{[32]}$.

Now we are passing through a very crucial time with a global pandemic. Globally all health authorities are now very concerned about the COVID-19, as it is the major task to assure the safety of the people. From the previous information, it is also true that just after the pandemic there will be several health issues that must be taken as serious. In many countries, it's already started to take action for future threats. However, the major three challenges those must be faced in very near future are the antibiotic resistance and gastrointestinal microbe related issues, management of NCDs and chronic diseases, and most importantly the Mental Health that is the result of long isolation, loosing of jobs, fear and panic of COVID-19. Without this, we have other issues like overuse of vitamins and notorious medicine recommended by unauthorized sources. These medicines are having side effects which can't be ignored.

\section{CONCLUSIONS}

COVID-19 has hampered our regular lives and stopped several industries temporarily because of lockdown or isolations and these results global economic instability. Natural or climate pollution has reduced in a significant number, which is good for the future but the two giant issues we have to face in nearer future are economic challenges and several previously concerning health problems those were remained unfocused for the pandemic crisis.

\section{CONTRIBUTION OF AUTHORS}

Research concept- Omer Fayshal Pavel

Research design- Omer Fayshal Pavel
Supervision- Dr. Md. Omar Faruk Parvez

Materials- Omer Fayshal Pavel, S. M. Shahnawaz Rashid

Data collection- Omer Fayshal Pavel

Data analysis and interpretation- Omer Fayshal Pavel, Dr. Md. Omar Faruk Parvez

Literature search- Omer Fayshal Pavel, S. M. Shahnawaz Rashid

Writing article- Omer Fayshal Pavel, Dr. Md. Omar Faruk Parvez, S. M. Shahnawaz Rashid

Critical review- Dr. Md. Omar Faruk Parvez

Article editing- Dr. Md. Omar Faruk Parvez, S. M. Shahnawaz Rashid

Final approval- Dr. Md. Omar Faruk Parvez

\section{REFERENCES}

[1] Alam MS, Alam MZ, Nazir KN, Bhuiyan MA. The emergence of novel coronavirus disease (COVID-19) in Bangladesh: Present status, challenges, and future management. J Adv Vet Anim Res., 2020; 7(2): 198208.

[2] Astuti I. Severe Acute Respiratory Syndrome Coronavirus 2 (SARS-CoV-2): An overview of viral structure and host response. Diabetes Metab Syndr., 2020; 4(14): 407-12.

[3] Huang C, Wang Y, Li X., Ren L, Zhao J., Hu Y., Zhang L, et al. Clinical features of patients infected with 2019 novel coronavirus in Wuhan, China. Lancet, 2020; 395(10223): 497-506.

[4] Song Y, Liu P, Shi XL, Chu YL, Zhang J, et al. SARS-CoV2 induced diarrhoea as onset symptom in patient with COVID-19. Gut., 2020; 69(6): 1143-44.

[5] Wong SH, Lu, RN, Sung JJ. Covid-19 and the digestive system. J. Gastroenterol Hepatol., 2020; 35(5): 74448.

[6] Zhang H, Kang Z, Gong H, Xu D, Wang J, et al. The digestive system is a potential route of 2019-nCov infection: a bioinformatics analysis based on singlecell transcriptomes. BioRxiv, 2020.

[7] Bavishi C, Maddox TM, Messerli FH. Coronavirus disease 2019 (COVID-19) infection and renin angiotensin system blockers. JAMA Cardiol., 2020.

[8] Li MY, Li L, Zhang Y, Wang XS. Expression of the SARS-CoV- 2 cell receptor gene ACE2 in a wide variety of human tissues. Infect Dis Poverty, 2020; 9: 1-7.

[9] Li Q, Guan X, Wu P, Wang X, Zhou L. et al. Early transmission dynamics in Wuhan, China, of novel coronavirus-infected pneumonia. N Engl J Med., 2020. 
[10]Worldometers. Coronavirus Update (Live). [Cited 2020 Aug 12], Available from https://www.worldometers.info/coronavirus/.

[11]World Health Organization (WHO). Coronavirus disease (COVID-19) advice for the public. [Cited 2020 Aug 12], Available from https://www.who.int/emergencies/diseases/novelcoronavirus-2019/advice-for-public.

[12]Guerriero C., Haines A., Pagano M. Health and sustainability in post-pandemic economic policies. Nat Sustain, 2020; pp. 1-3.

[13]Almond D. Is the 1918 influenza pandemic over? Long-term effects of in utero influenza exposure in the post-1940 US population. J. Polit. Econ, 2006; 114(4): 672-712.

[14]Sanidad KZ, Xiao H, Zhang G. Triclosan, a common antimicrobial ingredient, on gut microbiota and gut health. Gut Microbes, 2019; 10(3): 434-37.

[15]Jutkina J, Marathe NP, Flach CF, Larsson DGJ. Antibiotics and common antibacterial biocides stimulate horizontal transfer of resistance at low concentrations. Sci Total Environ., 2018; 616: 172-78.

[16]Bloomfield SF, Stanwell R., Crevel RWR, Pickup J. Too clean, or not too clean: the hygiene hypothesis and home hygiene. Clin Exp Allergy, 2006; 36(4): 402-25.

[17]Gerasimidis K, Bryden K, Chen X, Papachristou E, Verney et al. The impact of food additives, artificial sweeteners and domestic hygiene products on the human gut microbiome and its fibre fermentation capacity. Eur J Nutr., 2019; pp. 1-18.

[18]Yueh MF, Tukey RH. Triclosan: a widespread environmental toxicant with many biological effects. Annu Rev Pharmacol Toxicol., 2016; 56: 25172.

[19]Tun MH, Tun HM, Mahoney JJ, Konya TB, Guttmann DS, et al. Postnatal exposure to household disinfectants, infant gut microbiota and subsequent risk of overweight in children. CMAJ, 2018; 190(37): 1097-107.

[20]Donaghy JA, Jagadeesan B, Goodburn K, Grunwald L, Jensen $O N$, et al. Relationship of sanitizers, disinfectants, and cleaning agents with antimicrobial resistance. J Food Prot., 2019; 82(5): 889-902.

[21]Chapman JS. Disinfectant resistance mechanisms, cross-resistance, and co-resistance. Int. Biodeterior. Biodegrad., 2013; 51(4): 271-76. [22]lacucci M, Cannatelli R, Labarile N, Mao R, Panaccione $R$, et al. Endoscopy in inflammatory bowel diseases during the COVID-19 pandemic and post-pandemic period. Lancet Gastroenterol Hepatol., 2020; 5(6): 598-606.

[23]World Health Organization. COVID-19 significantly impacts health services for noncommunicable diseases. [Cited 2020 Aug 12], Available from https: https://www.who.int/news-room/detail/01-062020-covid-19-significantly-impacts-health-servicesfor-noncommunicable-diseases.

[24]Dyer O. Covid-19: Pandemic is having "severe" impact on non-communicable disease care, WHO survey finds, 2020.

[25]Alberti G. Noncommunicable diseases: tomorrow's pandemics. Bulletin of the World Health Organization, 2001; 79: 10.

[26] Galatzer IR, Huang SH, Bonanno GA. Trajectories of resilience and dysfunction following potential trauma: A review and statistical evaluation. Clin Psychol Rev., 2018; 63: 41-55.

[27]Taylor S, Asmundson GJ. Life in a post-pandemic world: What to expect of anxiety-related conditions and their treatment.J Anxiety Disord., 2020; 72: 102231.

[28]Taylor S, Landry C, Paluszek M, Fergus TA, McKay D et al. Development and initial validation of the COVID Stress Scales. J Anxiety Disord., 2020; 102232.

[29]Heeren A. On the Distinction between Fear and Anxiety in a (Post) Pandemic World: A Commentary on Schimmenti et al. Clin Neuropsych., 2020; 17(3): 189-91.

[30]Öhman A. Fear and anxiety: Overlaps and dissociations. Handbook of emotions, 2008, pp. 70928.

[31]Greenberg N, Docherty M, Gnanapragasam S, Wessely $\mathrm{S}$. Managing mental health challenges faced by healthcare workers during covid-19 pandemic. BMJ, 2020; 368.

[32] The National Institute for Health and Care Excellence, Post-traumatic stress disorder. [Cited 2020 Aug 12], Available from https://www.nice.org.uk/guidance/ng116/chapter/R ecommendations\#principles-of-care. 
[33] World Health Organization. WHO publishes list of bacteria for which new antibiotics are urgently needed [Cited 2020 Aug 16], Available from https://www.who.int/news-room/detail/27-022017-who-publishes-list-of-bacteria-for-which-newantibiotics-are-urgently-needed. 